\title{
Effect of Oscillatory Breathing on the Variability of the RR Intervals and its Prognostic Importance in Individuals with Left Ventricular Global Systolic Dysfunction
}

\author{
Paulo Roberto Benchimol Barbosa, José Barbosa Filho, Ivan Cordovil \\ Rio de Janeiro, RJ - Brazil
}

\begin{abstract}
Objective - To assess the effect of the oscillatory breathing on the variability of RR intervals $(V-R R)$ and on prognostic significance after one year follow-up in subjects with left ventricular global systolic dysfunction.

Methods - We studied 76 subjects, whose age ranged from 40 to 80 years, paired for age and gender, divided into two groups: group I - 34 healthy subjects; group II - 42 subjects with left ventricular global systolic dysfunction (ejection fraction $\leq 0.40$ ). The ECG signals were acquired during 600s in supine position, and analyzed the variation of the thoracic amplitude and the V-RR. Clinical and V-RR variables were applied into a logistic multivariate model to foretell survival after one year follow-up.
\end{abstract}

Results - Oscillatory breathing was detected in $35.7 \%$ of subjects in vigil state of group II, with a concentration of the spectral power in the very low frequency band, and was independent of the presence of diabetes, functional class, ejection fraction, cause of ventricular dysfunction and survival after one year follow-up. In the logistic regression model, ejection fraction was the only independent variable to predict survival.

Conclusion - 1) Oscillatory breathing pattern is frequent during wakefulness in the left ventricular global systolic dysfunction and concentrates spectral power in the very low band of $V-R R$;2) it does not relate to severity and cause of left ventricular dysfunction; 3) ejection fraction is the only independent predictive variable for survival in this group of subjects.

Key words: oscillatory breathing, heart failure, variability in the RR intervals

Instituto Nacional de Cardiologia Laranjeiras - MS - Universidade Gama Filho Mailing address: Paulo Roberto Benchimol Barbosa - Rua Pompeu Loureiro, 36/ 702 - 22061-000 - Rio de Janeiro, RJ, Brazil - e-mail:ecgar@ecgar.org English version by Stela Maris C. e Gandour
In healthy individuals, the duration of RR intervals in tha surface electrocardiogram varies cyclically from beat to beat. This variation is more significant in youngsters, is modulated by breathing movements, and is called respiratory sinus arrhythmia.

The characteristics of the breathing movements can alter the cyclic variations observed between successive electrocardiographic $\mathrm{R}$ waves (RR intervals). These alterations result both from direct influences on the modulation of the parasympathetic nervous system and the indirect influences of chemoreceptor mechanisms ${ }^{1}$.

In approximately $50 \%$ of the individuals with left ventricular global systolic dysfunction, the presence of abnormal patterns of breathing can be identified. In these individuals, oscillatory patterns of breathing (oscillatory breathing) can be observed both during sleep and during wakefulness, and are characterized by successive periods of tachy/hyperpnea followed by brady/hypopnea, and, eventually, apnea ${ }^{2-10}$.

Of the types of oscillatory breathing of clinical significance, Cheyne-Stokes respiration and periodic breathing stand out. Cheyne-Stokes respiration is the breathing pattern in which periods of tachy/hyperpnea are followed by regular periods of apnea. In periodic breathing, however, the periods of tachy/hyperpnea are cyclically followed by periods of brady/hypopnea with no apnea.

Although some authors have reported that oscillatory breathing, especially when observed during wakefulness, may be associated with more severe clinical findings and a more reserved prognosis ${ }^{2,6-8}$, the subject remains controversial.

This study aimed at analyzing the effects of oscillatory breathing on the behavior of the cyclic variations of the normal electrocardiographic RR intervals (V-RR) during wakefulness in individuals with left ventricular global systolic dysfunction and in sinus rhythm, and at assessing the influence of oscillatory breathing on clinical and laboratory findings, and on the mortality rate of the individuals studied. 


\section{Methods}

The study protocol was designed according to the principles of the Declaration of Helsinki and pertinent Brazilian legislation and was submitted to and approved by the Committee on Medical Ethics of the Instituto Nacional de Cardiologia Laranjeiras.

In the present study, 76 individuals of a consecutive cohort were included. They were referred for assessment in the sector of noninvasive electrocardiology of the Division of Arterial Hypertension of the Instituto Nacional de Cardiologia Laranjeiras from January 1998 to January 2001. These individuals belonged to the database of signal-averaged electrocardiographic signals, which was compiled in the same sector of the Instituto Nacional de Cardiologia Laranjeiras from September 1997 onwards. They were divided into two groups, adjusted by age, sex, and body mass index. Thirty-four individuals were healthy (group I) and 42 had left ventricular global systolic dysfunction (group II). All group II individuals were regularly taking diuretics, angiotensin-converting enzyme inhibitors, and digitalis, prescribed based on criteria of primary clinical evaluation. They were in sinus rhythm and their NYHA functional classes ranged from II to IV.

The individuals in each group were divided according to two age brackets as follows: subgroup a - from 40 to 59 years; subgroup b - from 60 to 80 years. Group I comprised 21 individuals between 40 and 59 years (group Ia - mean \pm standard deviation (SD) [median] -48.7 \pm 5.2 [48.0] years, 10 males), and 13 individuals between 60 and 80 years (group Ib $-71.3 \pm 5.9$ [71.0] years, 8 males). The clinical history, physical examination, laboratory tests, radiological examination, and electrocardiography at rest were normal in all group I individuals.

Group II comprised 18 individuals between 40 and 59 years (group IIa - 48.7 \pm 6.2 [48.0] years, 14 males) and 24 individuals between 60 and 80 years (group IIb $-69.3 \pm 5.7$ [69.0] years, 8 males).

All group II individuals had left ventricular ejection fraction $\leq 40 \%$ assessed on one-dimensional echocardiography. Twenty-two individuals had dilated heart disease of undetermined origin (cine coronary angiography with normal coronary arteries), 12 individuals had ischemic heart disease (previous history of myocardial infarction; presence of segmentary contractility changes on two-dimensional echocardiography; electrocardiographic changes compatible with necrosis in at least two leads exploring the same region and in accordance with the echocardiographic changes), and 8 individuals had hypertensive heart disease [myocardial mass index $(\mathrm{MMI})>130 \mathrm{~g} \cdot \mathrm{m}^{-2}$, and MMI $>150 \mathrm{~g} \cdot \mathrm{m}^{-2}$ for females assessed by the Devereux formula divided by the body surface area]. Six individuals had noninsulin-dependent diabetes mellitus (DM2) and kept glycemia under control with diet or oral antidiabetic drugs, or both. Smoking, alcoholic ingestion, and the degree of sedentary lifestyle were evaluated in the groups and showed no differences in their distribution.
All individuals underwent directed anamnesis, physical examination, and complementary tests (glycemia, cholesterolemia, renal and hepatic functions, electrocardiography at rest, chest radiography, and echocardiography). The following parameters were assessed: heart rate, respiratory rate and amplitude, body weight, and height. Blood pressure was measured in both arms with a mercury-column sphygmomanometer. The echocardiographic measurements were performed according to the protocols of the echocardiography department of the Instituto Nacional de Cardiologia Laranjeiras. The electrocardiographic signals were acquired for analyzing V-RR as described below. All consultations and examinations were performed from $10 \mathrm{AM}$ to $6 \mathrm{PM}$. In group II, 20 individuals were in functional class III-IV.

The electrocardiographic signals were taken during $600 \mathrm{~s}$, at a sampling rate of $1 \mathrm{kHz}$, with the A/D CAD-1232 converter of 14 bits coupled to the AECG03 amplifier (both Lynx Tecnologia Ltda., São Paulo, Brazil) and analyzed using the ECGAR ${ }^{\circledR}$ system ${ }^{11}$. The signals were acquired with the individuals lying in the dorsal decubitus position and breathing at their natural rate in an isolated environment at a constant temperature of $25^{\circ} \mathrm{C}$, during the period from 10 AM to 6 PM. The examinations were performed at intervals of at least 4 hours after the last meal, and in the smokers, at least 30 minutes after the last tobacco load.

The analysis of the breathing movements was performed with the individuals lying in the dorsal decubitus position on a tilt table and breathing spontaneously, and, after a 5-minute rest, capture of the signals was initiated. For the group II individuals who could not tolerate the dorsal decubitus position at zero degree, the table was tilted between 30 and 45 degrees, for the examination to be comfortably performed.

The respiratory rate and amplitude of the breathing movements were analyzed by using the protocol reported by Moody et al ${ }^{12}$, and the behavior of oxygen $\left(\mathrm{O}_{2}\right)$ saturation was analyzed by using external oximetry. Breathing was considered normal when the respiratory rate was between 12 and 24 breaths per minute, and the amplitude of breathing movements and $\mathrm{O}_{2}$ saturation varied little. The presence of oscillatory breathing was identified when the amplitude of breathing, the respiratory rate, and the values of $\mathrm{O}_{2}$ saturation varied cyclically, characterizing Cheyne-Stokes respiration and periodic breathing. The oscillatory pattern was confirmed with the graphic recording of the breathing movements.

All group I and II individuals had good quality electrocardiographic signals, which allowed an adequate evaluation of the breathing movements.

The electrocardiographic signals were analyzed in the frequency domain. The series of normal consecutive RR intervals detected on the surface electrocardiogram was linearly interpolated, and the power spectrum was calculated by using the rapid Fourier transform analysis according to the previously described technique ${ }^{13}$. Areas of the power spectrum were assessed according to very low frequency regions, defined between $0.003 \mathrm{~Hz}$ and $0.05 \mathrm{~Hz}$, low frequen- 
cy regions, between $0.05 \mathrm{~Hz}$ and $0.15 \mathrm{~Hz}$, and high frequency regions, between $0.15 \mathrm{~Hz}$ and $0.40 \mathrm{~Hz}$.

The variation in thoracic amplitude was analyzed through synchronous comparison with the cardiac beats, applying the function of quadratic spectral coherence $\left(\mathrm{r}^{2}\right)$ between both variables, and analysis of the values in the very low, low, and high frequency bands. The confidence intervals of $r^{2}$ were calculated by using a modified method reported by Miranda de Sá et al ${ }^{14}$. In practice, $\mathrm{r}^{2}$ values $>0.6$ were considered significant.

The variables of the frequency domain were presented in absolute values (very low frequency, low frequency, and high frequency) and percentages relative to the total power of modulation ( $\mathrm{pVLF}, \mathrm{pLF}$, and pHF, respectively), and expressed as means \pm SD. The absolute values were transformed into their natural logarithms (LnT) for normalization before the analysis, because of the strong asymmetry in its function of density of probability ${ }^{15,16}$.

The comparisons of the frequency domain variables, both in percentage and absolute values between groups I and II were performed by using the 2-tailed Student $t$ test for the same age brackets in each group. The study of the V-RR variables in different age brackets was carried out by Barbosa et al ${ }^{11,15}$ and Barbosa-Filho et al ${ }^{13}$, but was not performed in the present study. Analysis of the relative distribution of the power spectrum in the frequency bands was performed by using the $\chi^{2}$ adherence test against the hypothesis of uniform distribution of power. The variables expressing counting values were analyzed through the $\chi^{2}$ test with the Yates correction or the Fisher comparison for small samples.

For analyzing diagnostic significance in group II, the variables $\mathrm{pVLF}, \mathrm{pLF}$, and $\mathrm{pHF}$ were dichotomized between individuals with normal and oscillatory breathing according to their age bracket. For this, the method that analyzes the function defined by the product of the functions of probability distribution in each group was used. The values of specificity and sensitivity were identified at the points that maximize this function ${ }^{13}$. Odds ratio and the respective 95\% confidence intervals ( $95 \% \mathrm{CI}$ ) were calculated for each variable alone, and the statistical significances were evaluated through the $\chi^{2}$ test applied to the comparison of proportions.

The results were used for the construction of a multivariate logistic regression model aiming at analyzing the presence of variables with independent predictive values for survival after 1 year of evolution. The exploratory variables pHF, pLF, pVLF, DM2, left ventricular ejection fraction, functional class, and Cheyne-Stokes respiration were adjusted to the model to foretell survival and were selected by the stepwise process. Adjustment to the regression model was evaluated through the $\chi^{2}$ adherence test with 3 degrees of freedom, and the adjusted predictive variables were analyzed by using the $\chi^{2}$ test of maximal likelihood ratio. The odds ratio values for each variable were calculated after the best adjustment to the model with the respective $95 \%$ confidence intervals.

Data were analyzed with the statistical module Statgraphics 5.1 Plus (Statistical Graphics Corporation, USA) and MS Excel 2000 (Microsoft, USA). The alpha significance level was fixed in 0.05 for all tests.

\section{Results}

The values and distribution of the several frequency bands of the power spectrum in the groups studied are shown in table I. The absolute values of HF LnT, LF LnT, and VLF LnT in group II were significantly lower $(\mathrm{p}<0.001)$ than those observed in group I.

In regard to intragroup analysis, the values of HF LnT, LFLnT, and VLF LnT were significantly different between subgroups Ia and $\mathrm{Ib}(\mathrm{p}<0.001)$, but not between subgroups IIa and IIb.

\begin{tabular}{|c|c|c|c|c|c|c|}
\hline & G-Ia & G-IIa & $\mathrm{p}^{*}$ & $\mathrm{G}-\mathrm{Ib}$ & G-IIb & $\mathrm{p}^{*}$ \\
\hline $\mathrm{N}$ & 21 & 18 & & 13 & 24 & \\
\hline Age (years) $)^{* *}$ & $48.7 \pm 5.2[48]$ & $48.7 \pm 6.2[48]$ & NS & $71.3 \pm 5.9[71]$ & $69.3 \pm 5.7[69]$ & NS \\
\hline $\operatorname{Sex}(F / M)$ & $11 / 10$ & $4 / 14$ & NS & $5 / 8$ & $16 / 8$ & NS \\
\hline $\mathrm{SBP}(\mathrm{mm} \mathrm{Hg})$ & $125.0 \pm 10.0$ & $128.0 \pm 12.0$ & NS & $138.0 \pm 10.6$ & $130.0 \pm 13.0$ & NS \\
\hline $\mathrm{DBP}(\mathrm{mm} \mathrm{Hg})$ & $83.0 \pm 5.1$ & $78.0 \pm 6.0$ & NS & $80.0 \pm 5.4$ & $75.0 \pm 8.0$ & NS \\
\hline Glycemia $>120 \mathrm{mg} / \mathrm{dL}$ & 0 & 2 & - & 0 & 4 & - \\
\hline $\mathrm{BMI}>25 \mathrm{Kg} \times \mathrm{m}^{-2}$ & 0 & 0 & - & 0 & 0 & - \\
\hline $\mathrm{EF}<40 \%$ & 0 & 18 & - & 0 & 24 & - \\
\hline MHR (bpm) & $69.2 \pm 8.6$ & $84.5 \pm 14.0$ & NS & $71 \pm 111.4$ & $86.5 \pm 15$ & NS \\
\hline $\mathrm{HF} \operatorname{LnT}\left(\mathrm{LN} \mathrm{ms}{ }^{2}\right)$ & $5.8 \pm 1.0$ & $2.9 \pm 1.6$ & $<0.001$ & $4.4 \pm 1.0$ & $2.9 \pm 1.0$ & $<0.001$ \\
\hline $\operatorname{LF} \operatorname{LnT}\left(\operatorname{Ln} \mathrm{ms}^{2}\right)$ & $5.8 \pm 1.1$ & $3.3 \pm 1.7$ & $<0.001$ & $4.7 \pm 1.7$ & $3.1 \pm 1.1$ & $<0.001$ \\
\hline VLF LnT $\left.(\operatorname{Lnms})^{2}\right)$ & $5.9 \pm 0.7$ & $3.5 \pm 1.4$ & $<0.001$ & $5.2 \pm 1.0$ & $4.4 \pm 1.8$ & $<0.01$ \\
\hline $\mathrm{NB} / \mathrm{OB}$ & $21 / 0$ & $8 / 10$ & $<0.001$ & $13 / 0$ & $19 / 5$ & NS \\
\hline $\mathrm{pHF}(\%)$ & $28.1 \pm 14.2$ & $9.0 \pm 10.1$ & $<0.001$ & $21.0 \pm 13.7$ & $13.9 \pm 12.1$ & NS \\
\hline $\operatorname{pLF}(\%)$ & $34.1 \pm 18.7$ & $14.6 \pm 9.8$ & 0.05 & $28.8 \pm 12.8$ & $19.6 \pm 13.6$ & NS \\
\hline $\operatorname{pVLF}(\%)$ & $37.8 \pm 14.2$ & $76.3 \pm 15.1$ & $<0.001$ & $50.3 \pm 17.3$ & $66.3 \pm 18.7$ & 0.01 \\
\hline
\end{tabular}


The behavior of the variables $\mathrm{pVLF}, \mathrm{pLF}$, and $\mathrm{pHF}$ in group II depended on the breathing pattern. Therefore, in the 27 individuals with normal breathing, the distribution of power in the different bands was similar to that found in control individuals. For the 15 individuals with oscillatory breathing, Cheyne-Stokes respiration or periodic breathing, the power was concentrated in the very low frequency band (fig. 1).

In group II, considering individuals with and without DM2, the means of the following variables showed no statistically significant differences: V-RR (respectively, for $\mathrm{pHF} 10.4 \% \pm 10.2 \% \times 16.4 \% \pm 16.3 \%$; for $\mathrm{pLF} 18.3 \% \pm$ $11.9 \% \times 9 \% \pm 8 \%$; forpVLF71.1\% $\pm 16.6 \times 74.5 \% \pm 23.4 \%$ ), left ventricular ejection fraction (respectively, $28.8 \% \pm 10.5 \% \mathrm{x}$ $36.8 \% \pm 9.4 \%$ ), distribution of the individuals in functional class III-IV (respectively, 66\% x 50\%), individuals with oscillatory breathing (respectively, $41 \%$ x $50 \%$ ), and survival after 1 year of evolution (respectively, $55 \%$ x $76 \%$ ).

The analysis of $\mathrm{r}^{2}$ between V-RR and the variation in thoracic amplitude showed that, both in group I and group II with normal breathing, a significant coherence was observed in all bands [very low frequency $(\mathrm{p}<0.05)$ and high frequency $(\mathrm{p}<0.05)]$. In group II, the value of $\mathrm{r}^{2}$ was significant only in the individuals with oscillatory breathing, in whom the $\mathrm{V}-\mathrm{RR}$ power was concentrated in the very low frequency $(\mathrm{p}<0.05)$ (fig. 2$)$.

The analysis of the odds ratio showed that the respira-

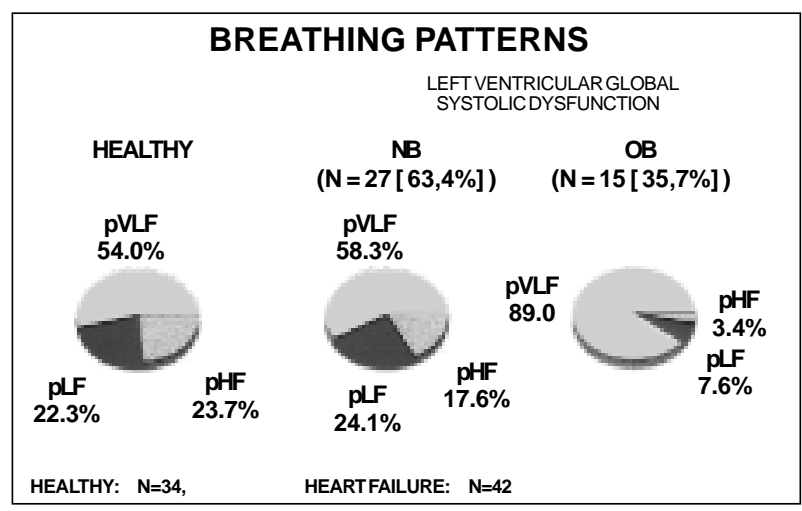

Fig. 1 - Percentage distribution of the frequency bands in the series of RR intervals in healthy individuals, in individuals with left ventricular global systolic dysfunction and normal breathing, and individuals with left ventricular global systolic dysfunction and oscillatory breathing. Note that in individuals with left ventricular global systolic dysfunction - oscillatory breathing (OB) - the modulating energy concentrates in the very-low-frequency band (pVLF). LVGSD = left ventricular global systolic dysfunction, $\mathrm{pVLF}=$ percentage of energy concentrated in the very-lowfrequency region, $\mathrm{NB}=$ normal breathing. tory types analyzed had no statistically significant association with the functional class or with the cause of the left ventricular global systolic dysfunction. In group II, a left ventricular ejection fraction $\leq 25 \%$ was associated with normal breathing with an odds ratio equal to 0.17 (95\% CI [0.03 to 0.88 ]; $\mathrm{p}=0.03$ ). The values for sensitivity and specificity are shown in table II. The variable $\mathrm{pVLF}$ in the cut off $>80 \%$ had a predictive accuracy of $100 \%$ and identified all individuals with the oscillatory breathing pattern. The comparisons of the clinical variables and V-RR between individuals with normal and oscillatory breathing are shown in table III.

Twelve months after the initial assessment, $42.2 \%$ of the group II individuals had died. Of these patients, in the initial assessment, $21.4 \%$ had oscillatory breathing and $78.6 \%$ had normal breathing (odds ratio $=0.34,95 \%$ CI [0.06 to 1.56$]$; NS), $78.6 \%$ were in functional class III-IV (odds ratio $=4.07 ; 95 \% \mathrm{CI}[0.71$ to 28.91$] ; \mathrm{NS}$ ), and $71.4 \%$ had left ventricular ejection fraction $\leq 25 \%$ (odds ratio $=7.00,95 \% \mathrm{CI}$ [1.21 to 43.91]; $\mathrm{p}=0.03$ ). The age and sex of these individuals who died after 12 months of evolution $(60.6 \pm 15.5$ years, $71.4 \%$ of males) did not significantly differ from those of the survivors in the same period $(59.8 \pm 12$ years, NS, $68.4 \%$ of males, NS). In the frequency domain, using the cut off values of pLF in $20 \%$ and of pHF in $15 \%$ to dichotomize the individuals who died and who survived, an odds ratio of 0.06 (95\% CI [0.01 to 0.48]; $\mathrm{p}=0.004$ ) was observed for $\mathrm{pLF}$ and an odds ratio of 0.38 (95\% CI [0.07 to 2.09]; NS) was observed for $\mathrm{pHF}$.

The logistic model to foretell survival after 1 year of evolution was adequately adjusted to the variables selected $\left(\chi_{3}^{2}=1.09 ; \mathrm{NS}\right)$. The variables DM2, functional class, and pVLF had no statistical significance in the model and were withdrawn during the process of stepwise selection, all with $\chi^{2}$ values $<0.01$ in the test of maximal likelihood ratio $(p \approx 0.99)$. The results of the adjusted model are shown in table IV.

\section{Discussion}

Respiratory disorders of the oscillatory breathing type are frequent in individuals with left ventricular global systolic dysfunction, especially those recorded with the individual awake. Some studies have shown that these disorders may act unfavorably on the prognosis of individuals with left ventricular global systolic dysfunction ${ }^{2,8,9,17}$.

When the electrocardiographic RR intervals are analyzed by using the power spectrum and recorded in

\begin{tabular}{|c|c|c|c|c|}
\hline & Cut off value & Specificity & Sensitivity & Total accuracy \\
\hline $\mathrm{pHF}(\%)$ & $>15 \%$ & $48.2 \% \quad(\mathrm{p}<0.01)$ & $100 \% \quad(\mathrm{p}<0.001)$ & $66.7 \% \quad(\mathrm{p}<0.001)$ \\
\hline $\mathrm{pLF}(\%)$ & $>20 \%$ & $59.3 \%(\mathrm{p}<0.01)$ & $100 \% \quad(\mathrm{p}<0.001)$ & $73.8 \% \quad(<0.001)$ \\
\hline pVLF $(\%)$ & $>80 \%$ & $100 \% \quad(\mathrm{p}<0.001)$ & $100 \%(\mathrm{p}<0.001)$ & $100 \% \quad(p<0.001)$ \\
\hline
\end{tabular}

$\mathrm{pHF}$ - percentage of energy of the power spectrum of V-RR concentrated in the high-frequency region; pLF - percentage of energy of the power spectrum of V-RR concentrated in the low-frequency region; pVLF - percentage of energy of the power spectrum of V-RR concentrated in the very-low-frequency region. 


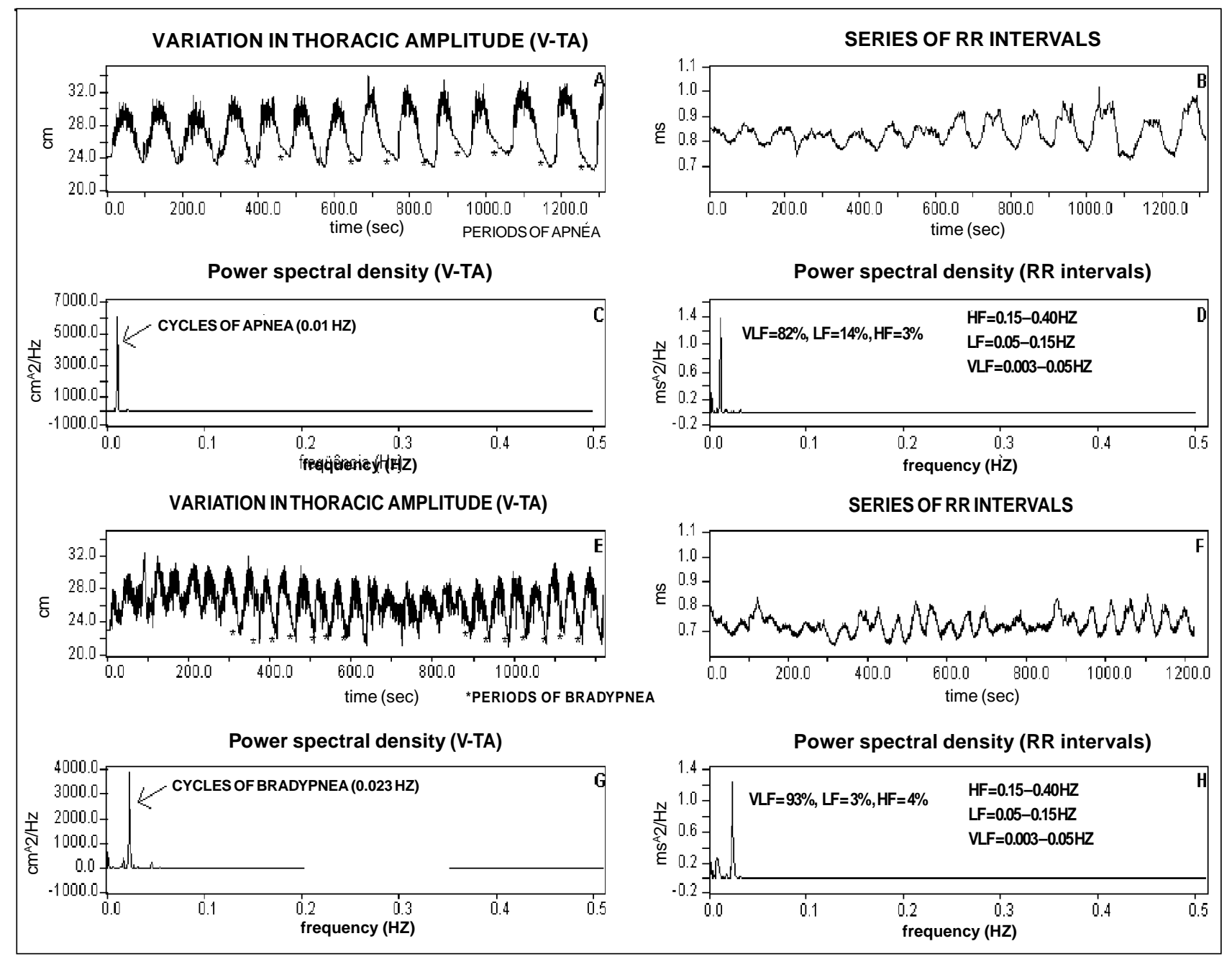

Fig. 2 - Simultaneous recording of the variation in thoracic amplitude and the RR intervals in individuals with Cheyne-Stokes respiration (A-B-C-D tracings) and periodic breathing (E-F-G-H tracings). Note, in both types of breathing, the spectral coherence in the VLF band (C-D tracings $\rightarrow r^{2}=0.72 ; G-H$ tracings $\rightarrow r^{2}=0.89$ ), between V-RR and V-TA. $\mathrm{V}-\mathrm{RR}=$ variability in the RR intervals. V-TA = variability in thoracic amplitude.

\begin{tabular}{|lccc|}
\hline \multicolumn{4}{|c|}{ Table III - Analysis of the breathing pattern in group II in regard to } \\
functional parameters *
\end{tabular}

different frequency bands, these intervals are observed to be modulated by respiration in a frequency band ranging from $0.01 \mathrm{~Hz}$ to $0.40 \mathrm{~Hz}$ (unpublished data). In that band, the effect of the breathing movements on the power spectrum of RR intervals can be predominantly felt by the cyclic variations in the tonus of the autonomic nervous system on the sinus node, mainly mediated by baroreflexes ${ }^{18}$.

Analyzing the behavior of the power spectrum of the RR intervals and comparing it with that observed in healthy individuals, two distinct patterns are observed in individuals with left ventricular global systolic dysfunction. In 15 (35.7\%) individuals with oscillatory breathing, a mean of $89.2 \%$ of the total spectral power was concentrated in the very low frequency band, despite having Cheyne-Stokes respiration or periodic breathing, showing that the RR intervals are mainly modulated by low frequency breathing movements (figs. 1 and 2). For the other 27 (64.3\%) individuals with no great cyclic variations in the amplitude of breathing movements, the behavior of the power spectrum had a normal distribution (fig. 1).

In the forms of oscillatory breathing described in the 


\begin{tabular}{|l|l|c|c|c|}
\hline \multicolumn{7}{|c|}{ Table IV - Results of the multivariate logistic regression model for predicting survival of group II individuals after 1 year of evolution with } \\
4 exploratory variables
\end{tabular}

literature, the energy reduction in the low and high frequency bands of the power spectrum, which represent the modulation of the efferent controls of the sympathetic-vagal system, has been attributed to the reduction in the regulatory capacity of the baroreflexes. This regulatory capacity may be due to the sensitivity loss in the peripheral baroreceptors or to the reduction in the rhythmicity of the efferent autonomic impulses originating in the cerebral nuclei ${ }^{19,20}$. This fact was very evident in the present study considering that the values of the energy in the high and low frequency bands were significantly lower in group II $(\mathrm{p}<0.001)$.

On the other hand, in left ventricular global systolic dysfunction, an increase in the sensitivity of the peripheral chemoreceptors and in the time of circulation between the lung and the regulatory cerebral breathing centers has been observed. Therefore, while the hypersensitivity of the chemoreceptors intensifies the response of the systems, which control gain (increased sensitivity to the alterations in $\mathrm{O}_{2}$ and $\mathrm{CO}_{2}$ ), and reduces the response of the systems that reduce it (a reduction in the $\mathrm{O}_{2}$ and $\mathrm{CO}_{2}$ reserves), the increase in the time of circulation delays the process of information transference. These alterations, on the other hand, cause instability of the feedback systems that control breathing, therefore determining the two forms of oscillatory breathing ${ }^{21-23}$. Considering that $\mathrm{V}-\mathrm{RR}$ is predominantly modulated by breathing movements, which, because of the already cited facts, are constituted by oscillations of very low frequency, the characteristics of the spectral power modulating V-RR are consistently modified. A deviation to the left in the frequency bands of the power spectrum is observed with a significant power concentration in the very low frequency band.

The importance of the recognition of these breathing types and of the V-RR behavior in left ventricular global systolic dysfunction during sleep and wakefulness, lies in the fact that they can change the quality of life and impair cardiopulmonary performance, making the prognosis of left ventri- cular global systolic dysfunction more reserved, regardless of the degree of impairment of clinical and laboratory parameters. These facts, especially those related to the development of arrhythmias and the deterioration of ventricular function could have as pathophysiological bases the autonomic imbalance, hypoxia, hypercapnia, and the increase in the sympathetic amines, therefore aggravating neurohormonal disorders, especially in regard to the elevated levels of catecholamines ${ }^{10,24}$. In regard to the clinical, laboratory, and evolutional findings of the individuals studied, the breathing patterns and the characteristics of V-RR were independent of functional class, the cause of left ventricular global systolic dysfunction, and the magnitude of the changes in the echocardiographic parameters. However, its arrhythmogenic capacity could not be assessed. Although other researchers reported that oscillatory breathing during wakefulness increases mortality in individuals with left ventricular global systolic dysfunction ${ }^{8}$, this fact was not observed in our study. Using a logistic regression model with the stepwise type of selection of the variables adjusted, the multivariate analysis showed that only left ventricular ejection fraction has an independent predictive value to foretell survival after a 1-year evolution $(\mathrm{p}=0.0005)$.

In conclusion: 1) oscillatory breathing is a common breathing type in individuals with left ventricular global systolic dysfunction observed in $35.7 \%$ of individuals with that disorder during wakefulness and characterized by a typical V-RR pattern; 2) oscillatory breathing has no correlation with the severity and cause of left ventricular global systolic dysfunction, the presence of DM2, and is not an independent predictive marker of mortality after 12 months of evolution; 3 ) the multivariate analysis reveals that the ejection fraction is the only variable with an independent predictive value for survival after a 1-year evolution in individuals with left ventricular global systolic dysfunction. 


\section{Referências}

1. Henry RA, Beightol LA, Eckber DL. Interaction between $\mathrm{CO}_{2}$ chemoreflexes and arterial baroreflexes. Am J Physiol Heart Circ Physiol 1998; 43: H2177-H87.

2. Ponikowski P, Anker SD, Chua TP, et al. Oscillatory breathing patterns during wakefulness in patients with chronic heart failure. Clinical implication and role of augmented peripheral chemosensitivity. Circulation 1999; 100: 2418-24.

3. Feld $\mathrm{H}$, Priest $\mathrm{S}$. A cyclic breathing pattern in patients with poor left ventricular function and compensated heart failure: a mild form of Cheyne-Stokes respiration? J Am Coll Cardiol 1993; 21: 971-4.

4. Mortara A, Sleight P, Pinna GD, et al. Abnormal awake respiratory patterns are common in chronic heart failure may prevent evaluation of autonomic tone by measures of heart rate variability. Circulation 1997; 96: 246-52.

5. Pinna GD, Maestri R, Montara A, La Rovere MT. Cardiorespiratory interaction during periodic breathing in awake chronic heart failure patients. Am J Physiol Heart Circ Physiol 2000; 278: H932-H41.

6. Tkacova R, Niroumand M, Lorenzi-Filho G, BradleyTD. Overnight shift from obstructive to central apneas in patients with heart failure. Role of $\mathrm{PCO}_{2}$ and circulatory delay. Circulation 2001; 103: 238-43.

7. Lorenzi-Filho G, Rankin F, Bies I, et al. Effects of inhaled carbon dioxide and oxygen on Cheyne-Stokes respiration in patients with heart failure. Am J Respir Crit Care Med 1999; 159: 1490-8.

8. Andreas S, Hagenah G, Möller C, et al. Cheyne-Stokes respiration and prognosis in congestive heart failure. Am J Cardiol 1996; 78: 1260-4

9. Montara A, Bernardi L, Pinna GD, et al. Alteration of breathing in chronic heart failure: clinical relevance of arterial oxygen saturation instability. Clin Sci (Colch) 1996; 91: 72-4.

10. Davies SW, John LM, Wedzicha JA, Lipkin DP. Overnight studies in severe chronic heart failure: arrhythmias and oxygen desaturation. Br Heart J 1991; 65: 77-83.

11. Barbosa PR, Barbosa Filho J, Morais de SáCA. Influência da idade, sexoe doença coronária sobre a modulação autonômica do coração. Arq Bras Cardiol 1996; 67: $325-9$
12. Moody GB, Mark RG, Zoccola A, Mantero S. Derivation of respiratory signals from multi-lead ECGs. Computers in Cardiology 1985; 12: 113-6.

13. Barbosa-Filho J, Barbosa PR, Cordovil I. Modulação Autonômica do Coração na Hipertensão Arterial Sistêmica. Arq Bras Cardiol 2002; 78: 181-8.

14. Miranda de Sá AMFL, Infantosi AFC, Simpson DM. A statistical technique for measuring synchronism between cortical regions in the EEG during rhythmic stimulation. IEEE Trans Biomed Eng 2001; 48: 1211-5.

15. Barbosa PR, Barbosa Filho J, Morais de Sá CA. Efeito da idade sobre a modulação autonômica do coração. Revista da SOCERJ 1995; 4: 79-84.

16. Bigger JTJr,Fleiss JL, Steiman RC, et al. Frequency domain measures of heart rate variability and mortality after myocardial infarction. Circulation 1992; 85: 164-71.

17. Hanly PJ, Zuberi-Khokhar NS. Increased mortality associated with CheyneStokes respiration in patients with congestive heart failure. Am J Resp Crit Care Med 1996; 153: 272-6.

18. Piepoli M, Sleight $P$, Leuzzi S, et al. Origin of respiratory sinus arrhythmias in conscious humans. An important role of arterial carotid baroreceptor. Circulation 1997; 95: 1813-21.

19. Saul JP, Arai Y, Berger RD, et al. Assessment of autonomic regulation in chronic congestive heart failure by heart rate spectral analysis. Am J Cardiol 1988 61: 1292-9.

20. Van de Borne P, Montano N, Pagani M, et al. Absence of low-frequency variability of sympathetic nerve activity in severe heart failure. Circulation 1997; 95: 1449-54.

21. Lahiri S, Hsiao C, Zhang R, et al. Peripheral chemoreceptors in respiratory oscillations. J Appl Physiol 1985; 58: 1901-8.

22. Khoo MC, Kronauer RE, Strohl KP, Slutsky AS. Factors inducing periodic breathing in humans: a general model. J Appl Physiol 1982; 53: 644-59.

23. Naughton M, Benard D, Tam A, et al. Role of hyperventilation in the pathogenesis of central sleep apneas in patients with congestive heart failure. Am Rev Respir Dis 1993; 148: 330-8.

24. Naughton MT, Benard DC, Liu PP, et al. Effects of nasal CPAP on sympathetic activity in patients with heart failure. Am J Respir Crit Care Med 1995; 152: 473-9. 